\title{
A Method to Integrate Geological Knowledge in Variogram Modeling of Facies: A Case Study of a Fluvial Deltaic Reservoir
}

\author{
Margaret Akoth Oloo, Congjiao Xie* \\ Faculty of Earth Resources, Key Laboratory of Tectonics and Petroleum Resources, China University of Geosciences, Wuhan \\ China \\ Email: a.maggarett@yahoo.com, *cxie2004@cug.edu.cn
}

How to cite this paper: Oloo, M.A. and Xie, C.J. (2018) A Method to Integrate Geological Knowledge in Variogram Modeling of Facies: A Case Study of a Fluvial Deltaic Reservoir. International Journal of Geosciences, 9, 337-353.

https://doi.org/10.4236/ijg.2018.96021

Received: May 7, 2018

Accepted: June 23, 2018

Published: June 26, 2018

Copyright $\odot 2018$ by authors and Scientific Research Publishing Inc. This work is licensed under the Creative Commons Attribution International License (CC BY 4.0).

http://creativecommons.org/licenses/by/4.0/

(c) (i) Open Access

\begin{abstract}
Variograms are important tools in the spatial distribution of facies and petrophysical properties. Due to the scarcity of subsurface well data, both spatially and quantity wise, variograms representing the data tend to have a lot of uncertainties. In order to reduce uncertainty in variograms, well data can be supplemented with the geological knowledge of the reservoir. This has been demonstrated by various authors in previous works. In their paper "Methodology to Incorporate Geological Knowledge in Variogram Modeling," A. Bahar and M. Kelkar introduced a methodology to incorporate geological knowledge by studying the energy level of the depositional environment and grain texture. They used these two attributes to determine the relative distance of continuity of the lithofacies and incorporated it in the variogram modeling. In this paper, we introduce another attribute that determines the continuity of lithofacies; the accommodation or deposition space. For illustration purpose, two sets of facies models were constructed: The first using subsurface well data only and the second using well data and geological information of the reservoir. The two sets of models showed significant variation in the property distribution. The first set gave a more random appearance of the facies distribution while the second set gave a more realistic depiction of the depositional environment of the reservoir. We concluded that other than the grain size and the energy level of the depositional environment, another important determinant for continuity in variograms is the knowledge of the depositional space. Incorporating the knowledge of the depositional environment enabled a more accurate estimation of the variogram parameters. This resulted in an improvement in the accuracy of the model.
\end{abstract}

\section{Keywords}

Geological Knowledge, Depositional Environment, Energy Level, Variogram 


\section{Introduction}

A reservoir model is a representation of a reservoir's characteristics (structure and properties). However, reservoir models do not accurately portray the subsurface reservoirs. This is due to the fact that a lot of estimation is done during the propagation of data in areas where data was not sampled. The subsurface data collected is usually not sufficient for meticulous reservoir modeling. This is especially true in the presence of a few vertical wells [1].

Accuracy of reservoir models is important in the petroleum industry as they have many functions. They include:

- Representation of different kinds of data (2D and 3D seismic, 4D time-lapse seismic, directly sampled rock formations in form of cuttings and cores, well logs) in a common format.

- Estimation of the original volumes of hydrocarbons.

- Simulating reservoir fluid flow behavior.

- Optimal placement of wells.

- Economic assessment of the reservoir.

- Establishment of appropriate field development scenarios.

- Uncertainty analysis [2].

The main steps involved in reservoir modeling include: building the structural design of the reservoir, stratigraphy modeling, facies modeling within each stratigraphic layer and petrophysical properties modeling based on the facies. This is a sequential approach work flow, where large scale properties are modeled first, followed by smaller uncertain properties. In the case of stochastic modeling, multiple realizations of the model are created in order to draw statistical inferences from the set of all outcomes. Modeling steps may however vary depending on the type of data available, time, reservoir type and human expertise.

In order to improve the accuracy of reservoir models, the subsurface data can be supplemented with other types of data, for example: outcrops data, the knowledge of the geological environment of the reservoir, analogue data and present day sedimentary environments which enable the enacting of former environments. These data can help in creating reservoir models that give a clearer representation of the subsurface.

In their paper, A. Bahar and M. Kelkar (2001) propose a method to incorporate geological knowledge by studying the energy level of the depositional environment at the time of lithofacies deposition. The procedure they came up with is as follows:

1) Infer geological knowledge.

2) Estimate conditioning variogram using hard data; apply the averaging technique for horizontal variogram when necessary.

3) Model the conditioning variogram considering the geological information as the soft information.

In their paper, the methodology was inferred by considering the energy level of the environment. The general rule being that a low energy environment will 
lead to a prolonged continuity compared to a high energy environment [3]. Based on the above criteria, the following depositional environments were classified in Table 1.

In addition to the energy level of the depositional environments, they also considered the roundness, sorting and size of the grains as an indication of the type of depositional environment as shown in Table 2. Whereby good grain sorting was an indication of a low energy environment hence longer continuity and poor sorting implicated a higher energy level, thus, a shorter continuity. Increase in grain roundness suggested an increase in wave or current intensity.

Using the two classifications above (Energy level and Grain texture and sorting), Bahar and Kelkar determined the relative distance of continuity of the lithofacies and incorporated it in variogram modeling. They hence concluded that including the knowledge of the depositional environment should always be part of reservoir modeling process.

In this paper, we expound more on the aspect of the knowledge of the depositional environment. Rather than only considering the energy level and grain size as a determinant of distance of continuity, other variables are considered. They include the deposition space available and the geometry of the depositional feature.

In summary, this paper defines a variogram, talks about the problem encountered in variogram modeling and reduces the uncertainty in variogram modeling by supplementing the sampled data with the geological knowledge of the reservoir. The knowledge of the reservoir is inferred by considering the energy level, deposition space and the geometry of the depositional feature. The geological knowledge gave information about the range and direction of the reservoir. A case study of facies modeling is done to illustrate the incorporation of the knowledge of the depositional environment in variogram modeling.

\subsection{Variograms}

\subsubsection{Variogram Definitions}

A lot of studies have been done on variograms and some of the definitions put forward include:

$A$ variogram is a description of the spatial continuity of the data [4].

Variograms are tools used to characterize the spatial variability of geological properties [5].

Table 1. Classification of depositional environments based on energy level according to A. Bahar and M. Kelkar [3].

\begin{tabular}{crr}
\hline Depositional environment & Energy level & Continuity \\
\hline Lagoon & High energy environment & Short continuity \\
Bays & Medium energy environment & Medium continuity \\
Restricted shelf & Medium to low energy environment & Medium to long continuity \\
Open shelf & Low energy environment & Long continuity \\
\hline
\end{tabular}


Table 2. Classification of grain type based to energy level according to A. Bahar and M. Kelkar [3].

\begin{tabular}{cc}
\hline Type of grain & Energy level \\
\hline Mudstones and Wackestone & Low \\
Packstone & Intermediate \\
Grainstone & High \\
\hline
\end{tabular}

The variogram is the expected squared difference between two data values separated by a distance vector [1].

Variograms are the central tool of Geostatistics which quantitatively express spatially corelated variation [6].

In a nutshell, a variogram is a model portraying the geological difference in a reservoir as a function of distance. The variogram is the primary tool in geostatistical analysis. Geological phenomena have the characteristic of spatial variation. Data collected close to each other will be similar and those collected further from each other will not be similar. Beyond a certain distance, there is no similarity at all [3]. Variograms are important tools in predicting spatial variation of geological phenomena in areas where we are not able to get access to the data [3]. In reservoir modeling, variograms are used in the simulation of reservoir properties such as facies, porosity, permeability and water saturation. The geostatistical algorithms used in reservoir modeling; sequential indicator simulation, sequential Gaussian simulation, and truncated Gaussian simulation require a variogram model for simulation of the reservoir properties. Variograms are hence vital in the modeling of reservoir properties and the flow behavior of the model [1]. Geological trends within the data must be removed before a variogram is modeled. This is because geostatistical algorithms work best on stationary data [1].

In the modeling of reservoir properties, our knowledge of the geometry and continuity of the different properties is portrayed by the variogram. The modelled properties eventually affect the reservoir's flow behavior or simulation studies and development decisions [1]. An interpretation of the variogram in all directions (3D) is necessary for full description of the geological continuities, which should be consistent with principles of geological deposition [1]. The variogram model should hence account for the sampled data, geological interpretation and analogue data [1]. Proper modeling of experimental variograms leads to a great improvement of reservoir models [1]. Reservoir simulation studies can be used to quantify the importance of variogram models [1].

A variogram as displayed in Figure 1 is basically a display of the mean square difference between two values as a function of their increment [7]. Data collected from the field is used to calculate the experimental variograms [8]. Matheron, 1965 described how to calculate the experimental variogram in the following equation.

$$
\gamma(h)=\frac{\sum_{i=1}^{n_{h}}\left(x_{i}-x_{i+h}\right)^{2}}{2 n_{h}}
$$




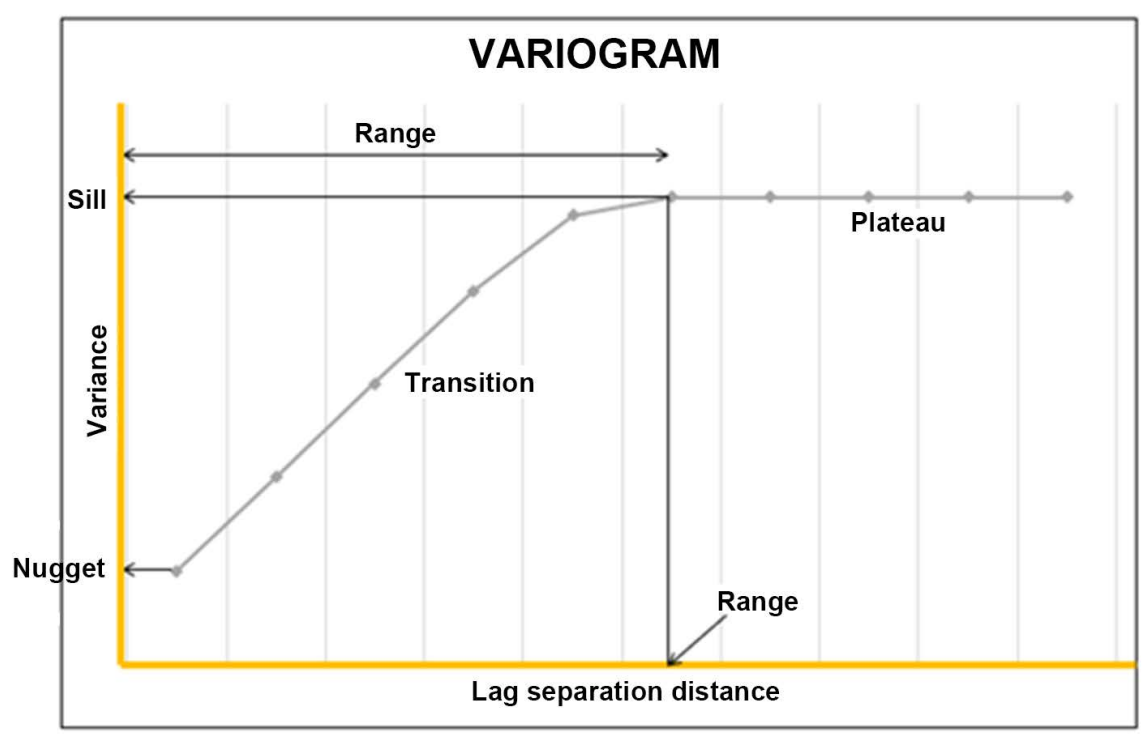

Figure 1. A variogram displaying variability between data points as a function of distance [2].

where, $\gamma(h)$ is a semi variogram,

$2 \gamma(h)$ is variogram,

$n_{h}$ is the number of pairs of points with the distance $h$ from each other participating in the variogram,

$x_{i}$ is a grade in point $i$,

$x_{i+h}$ is grade in a point with distance $h$ from the point $i$.

The terms variogram and semi variogram may be used interchangeably.

From Figure 1, we can see that the shorter the distance, the greater the correlation and the longer the distance, the less the correlation until ultimately, there is no more correlation between the data points.

The following parameters define a variogram; Range, sill and nugget.

\subsubsection{Characteristics of the Variogram}

Sill: The semi variance value at which the variogram levels off. Data is no longer auto correlated, also used to refer to the "amplitude" of a certain component of the semi variogram.

Range: The lag distance at which the semi variogram (or semi variogram component) reaches the sill value. The range in each direction captures the extent over which the geological property spreads [1].

Nugget: The semi variance where the separation distance is zero.

Plateau: The part of the variogram model where an increase in separation distance does not change the variogram value.

\subsubsection{Variogram Behavior}

Gringarten and Deutsch (2003) outlined the behaviors of variograms based on the geological variations of reservoir properties in their paper "Methodology for Improved Variogram Interpretation and Modeling for Petroleum Reservoir 
Characterization. Variogram behavior usually relates to the geology of the reservoir. The variogram behaviors they outlined in their paper include; Lack of spatial correlation, decreasing spatial correlation with distance, Geological trends, Areal trends, Stratigraphic layering and Geologic cyclicity [1].

\subsubsection{Difficulties in Variogram Modeling}

The biggest challenge in modeling variograms is the calculation of the horizontal variogram. In reservoir modeling, we need to create both vertical and horizontal variograms for spatial distribution in the vertical and horizontal direction respectively. Vertical variograms tend to be accurate due to availability of data in the wells. There is hence data correlation that can be computed to create the experimental variogram. Horizontal variograms, on the other hand are difficult to compute. This is as a result of the sparse nature of the subsurface data available of the reservoir as shown in Figure 2. There is hence uncertainty in the variograms [9]. In some cases, there is no correlation at any lag distance leading to nugget variograms. These variograms do not depict the true geological nature of the reservoir as they result in random distribution of the property. Horizontal variograms are more of an assumption than a true representation of the reservoir. Margaret Oliver et al. (2009) stated that taking a large sample can help mitigate the uncertainty. Large sample numbers and short lags increase the accuracy of the variograms as illustrated in figure two [6]. The uncertainty can also be reduced by incorporating the geological knowledge of the reservoir [3]. Vertical variograms are usually modelled first due to their higher levels of accuracy. The defined nugget, sill and variogram type can then apply to the horizontal variogram. The range of the horizontal variogram can be determined from additional knowledge of the depositional environment [3].

\subsubsection{Estimating the Variogram}

For any given lag, the semi variance, in one, two or three dimensions is calculated from the collected data [6]. The variogram should be estimated in different

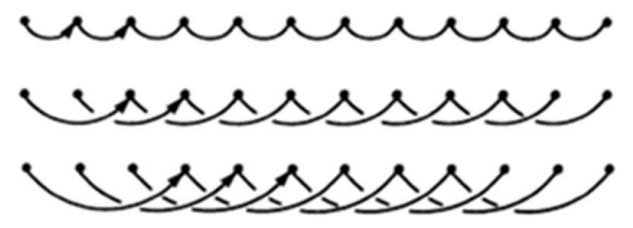

$\operatorname{lag} 1$

$\operatorname{lag} 2$

$\operatorname{lag} 3$

(a)

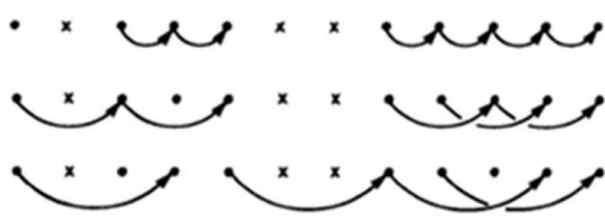

$\operatorname{lag} 1$

$\operatorname{lag} 2$

lag 3

(b)

Figure 2. (a) Complete data set, and (b) Incomplete data set, missing data represented by $\mathrm{x}$ showing sparse data in the horizontal direction [6]. 
directions to detect the difference property variation in all directions [6]. The collected sample values are used to calculate corresponding experimental variograms. The experimental variogram, when plotted displays the average change of a property with changing lag [6].

The experimental model cannot directly be used in the modeling process. An important step in geostatistical modeling is fitting the theoretical model to the experimental model [8]. A model variogram has to be fit to the samples to represent the true variogram of the study region [6]. Procedures are available for selecting and fitting theoretical models to experimental models using computer programs such as MLP and GenStat [6]. Before modern computers, semi variograms were fitted visually [10] i.e. fitting with the eye. McBratney and Webster (1986) did not recommend the method as they felt it was inadequate [11]. Oliver et al. (2009) buttressed their decision in Geostatistics in physical geography. Of recent, statisticians used variants of maximum likelihood to fit the models. Currently, most physical science researchers use variants of least square approaches to fit the theoretical variograms [10]. Cressie, 1985; McBratney and Webster, 1986 recommended fitting models by weighted least squares approximation as the reliable method and it is the one most widely used.

\subsubsection{Importance of Variograms}

- Geostatistical mapping and modeling algorithms require variogram models [1].

- Variograms help in the investigation and quantification of the spatial variability of geological phenomenon [1].

- Variograms summarize the spatial variation in the region of interest [6].

- Variograms quantify the spatial relationship of reservoir properties such as; facies, porosity, and permeability [3].

- Variograms affect the flow behavior of reservoir models [1].

- Variograms help to supplement the large scale seismic data at a smaller scale [1].

In summary, variograms portray our comprehension of the reservoir in terms of its geometry and continuity [3]. Accurate knowledge of the geology of the reservoir is hence a prerequisite of any variogram modeling study.

\subsection{Incorporating the Knowledge of the Depositional Environment in Variogram Modeling}

Geological knowledge is incorporated in variogram analysis to help reduce uncertainty. Well data is usually scarce spatially and quantity wise hence making it difficult to construct accurate models. The knowledge of the depositional environment helps to supplement the well data and give more information that we cannot extract from the well data.

\subsubsection{Depositional Environment}

Deposition is a geological process by which sediments are added to form a land mass. A depositional environment is a geomorphic setting in which a particular 
set of physical, chemical, and biological processes operate to generate a certain kind of sedimentary deposit. Depositional environments have both a static and dynamic element. The static elements include; basin geometry, sediment composition, water depth etc., while the dynamic elements include currents (wind, water), precipitation, climate [12]. Some of the static elements change eventually over geologic time, for example basin shapes [13]. However, the time period usually is extremely long.

Depositional environments are divided into three major categories as illustrated in Figure 3; Continental/Terrestrial (land) environments, Transitional /Marginal marine (coastal) environments and Marine (ocean) environments. Sediments accumulate in these environments to form depositional landforms.

Continental depositional environments; Continental depositional environments are depositional environments where by the sediments are deposited on the land or lakes. Examples of continental depositional environments include fluvial, desert, lacustrine, and glacial valleys [13].

Marginal marine depositional environments; Marginal marine environments lie along the boundary between continental and marine depositional systems. They include deltas, estuaries, beaches, barrier island, tidal flat.

Marine depositional environments; Marine depositional environments lie in the ocean deep. They include Continental shelf, continental slope, deep marine deposition (Table 3).

The sedimentary deposits outlined above are usually named after the process responsible for forming them. Rains and rivers produce fluvial deposits, lakes produce lacustrine deposits, ice produces glacial deposits and the sea produces marine deposits [14].

Most of the depositional environments outlined above are potential locations for reservoirs. During the deposition of the sediments, structures are formed that are indicative of the conditions in which they were created. Some of the conditions include; fluvial discharge, depth of the water, sediment (volume, grain size), wave climate, tidal range, current speed and the environmental setting. We will talk briefly about the fluvial depositional environments and deltas.

\subsubsection{Fluvial Depositional Environments}

Rivers are important sediment transportation channels to lakes and seas. However, deposition of sediments also occurs within the river course [13]. Rivers deposit sediments along their entire course whenever there is a reduction of depth or velocity [14]. This can occur when a river enters a shallow area or a large water body. Fluvial depositional features are formed by activities associated with rivers. Table four shows different depositional features formed by rivers, all of which can eventually form reservoirs.

\subsubsection{Fluvial Depositional Features}

Flood plains and levees: Most rivers are flanked by moderately flat land called a flood plains; Figure 4. Flood plains are formed by debris deposited when a river 


\section{Depositional Environments}

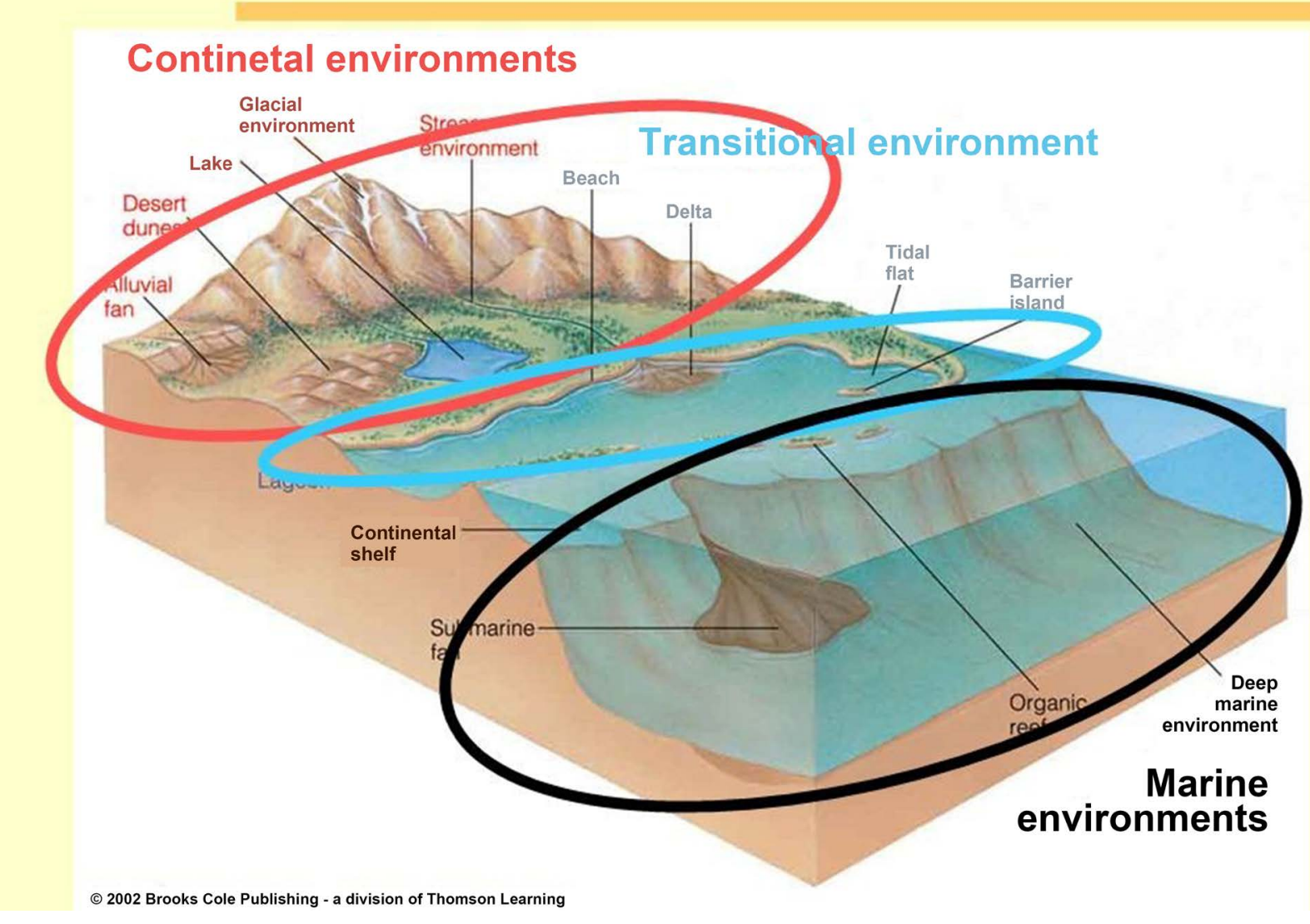

Figure 3. Classification of depositional environments.

Table 3. Classification of depositional environments.

\begin{tabular}{c|c|c}
\hline \multicolumn{2}{c}{ Depositional environments } \\
\hline Continental environments & Transitional environments & Marine environments \\
\hline Fluvial & Deltas & Continental shelf \\
Desert & Estuaries & Continental slope \\
Lacustrine & Beaches & \\
Glacial & Barrier islands & \\
& Tidal flat & \\
\hline
\end{tabular}

floods [14]. During flooding, the water rises beyond its banks and flows into the flood plain. Heavy and large sediments are deposited close to the river channel forming levees whereas fine sediments are deposited further forming flood plains. Floodplains are present along both braided and meandering rivers [13].

Delta: The word delta was used by the Greek philosopher Herodotus about 490 B.C. to describe the triangular-shaped alluvial plain formed at the mouth of the Nile River by deposits of the Nile distributaries [13]. As rivers enter large water bodies like lakes and oceans, the water slows down. Due to loss of kinetic 


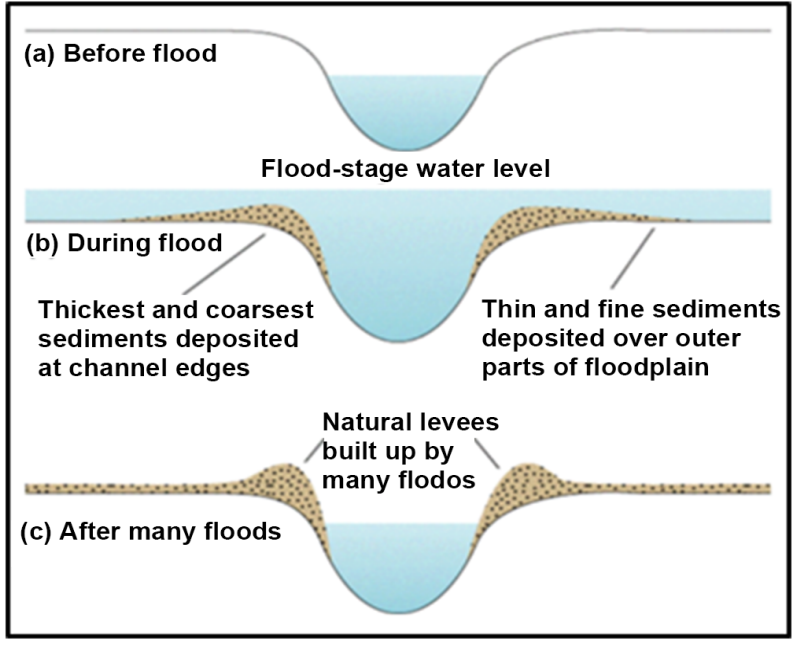

Figure 4. Floodplains and levees.

energy of the fluid, deposition occurs, building up layers of sediment on the sea bed as shown in Figure 5. The sediments eventually rise above the sea level and block the river mouth forming a delta. The coarse sediment is deposited near the river mouth and the finer sediments are carried seaward into deeper water.

Braided channels: These are mainly formed in the lower course of the river when the sediment load is very heavy and the water velovity is low. The stream deposits the sediments within the channel forming little bar like shaped islands as shown in Figure 6.

Point bars: In the meandering stage of the rivers, erosion occurs on the outer part of the meander, forming a cut bank, while deposition occurs on the inner part of the meander forming point bars; Figure 7. Point bars are attached to the river bank. The basic dynamics of flow around meanders leads to erosion on the outside parts of bends and deposition on the point bars [13].

Alluvial fans; Alluvial fans are cone shaped sedimentary bodies that are formed when rivers flow out of mountainous areas into a flat plane [14] as shown in Figure 8 . They tend to be coarse-grained at their mouths and relatively fine-grained at their edges [15].

Figure 9 shows a rivers lifecycle which is a main depositional environment for reservoirs, while Figure 10 shows the different factors affecting the speed of the river. The shape, geometry and size of a reservoir depend on the depositional environment in which it is formed. Using the knowledge of the depositional environment, we are able to determine the range, major and minor directions of continuity. In areas of low energy, there is less disturbance, hence the range of distribution of the sediments is longer. In areas of high amount of energy, the sediment particles are more disturbed hence their range will be shorter.

Another important factor that determines the continuity is the depositional space available during deposition. Take for example an alluvial fan; Figure 11. Alluvial fans are deposited as a stream flows down from a mountain, hence at a high energy level. However, due to the larger depositional space, the continuity 


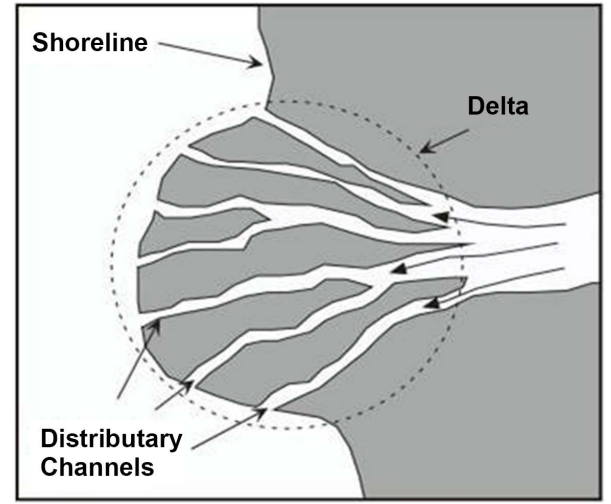

Figure 5. Delta.

Braided Channel

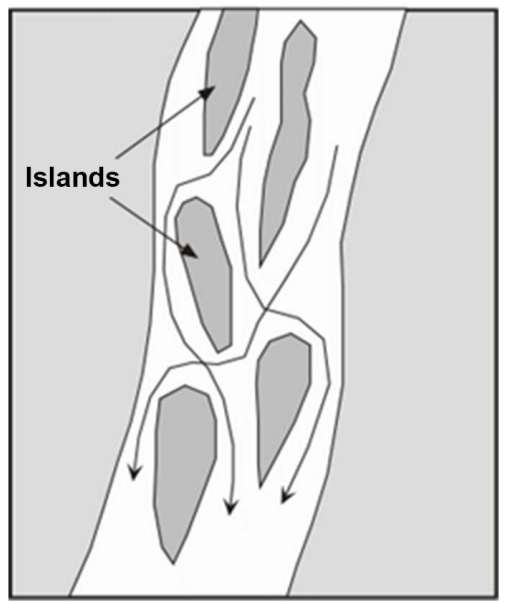

Figure 6. Braided channel.

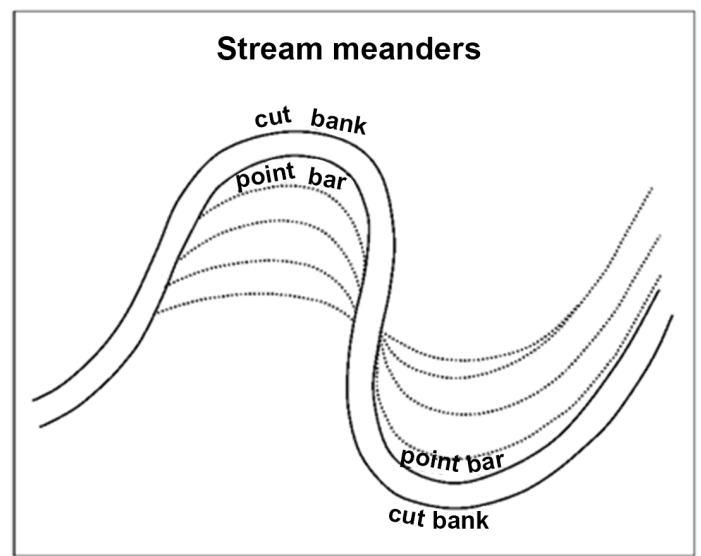

Figure 7. Point bar.

of the lithofacies will be long. The energy level of the depositional environment should hence not be the final determinant of continuity of lithofacies. The depositional space should be put into consideration as well. Table 4 shows different depositional features formed by rivers and their depositional space. 


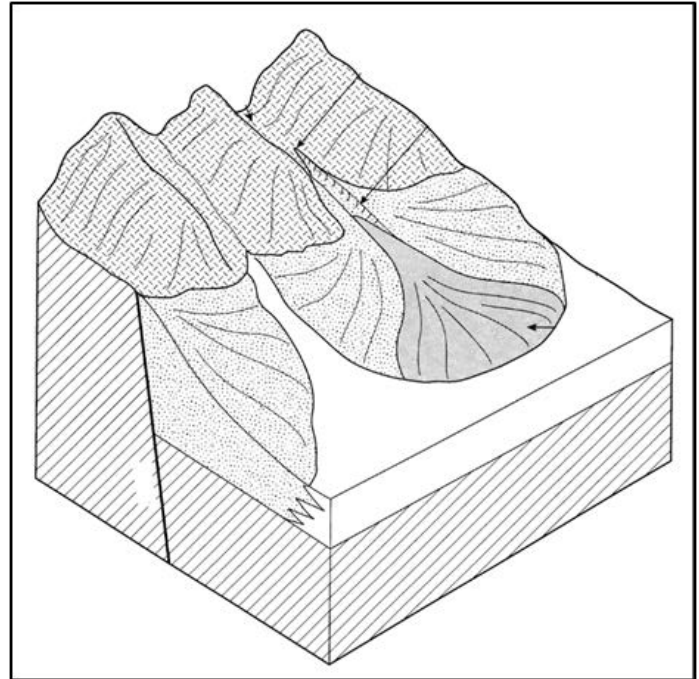

Figure 8. Alluvial fan.

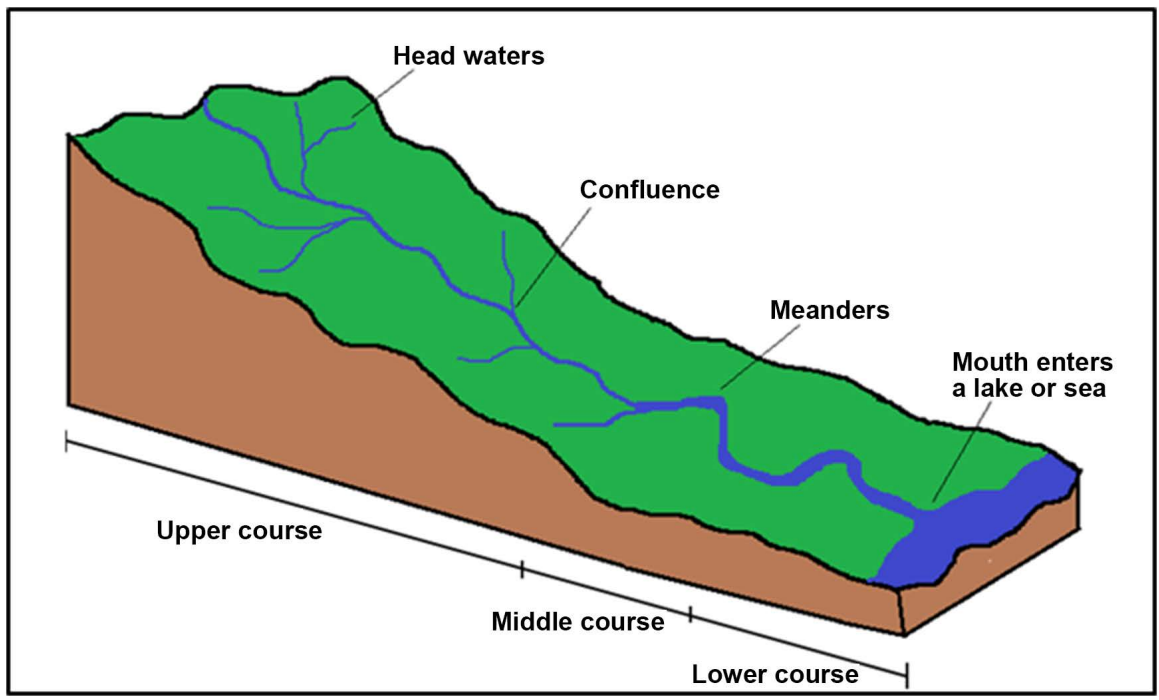

Figure 9. Lifecycle of a river.
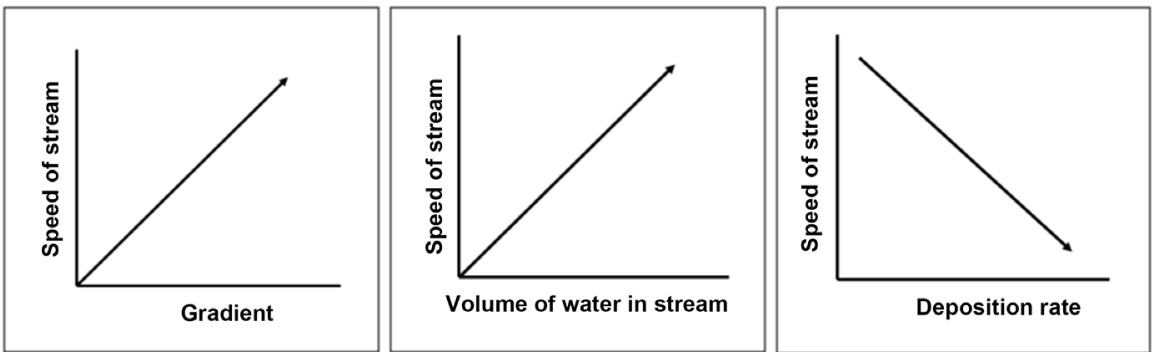

Figure 10. Speed of stream against gradient, stream volume and deposition rate.

\subsection{Case Study}

A case study is done using real field data. The reservoir under study is a fluvial delta reservoir. The field is a green field and has sparse well data. The horizontal variograms therefore carry a large amount of uncertainty. Information on the 


\section{Depositional environments}

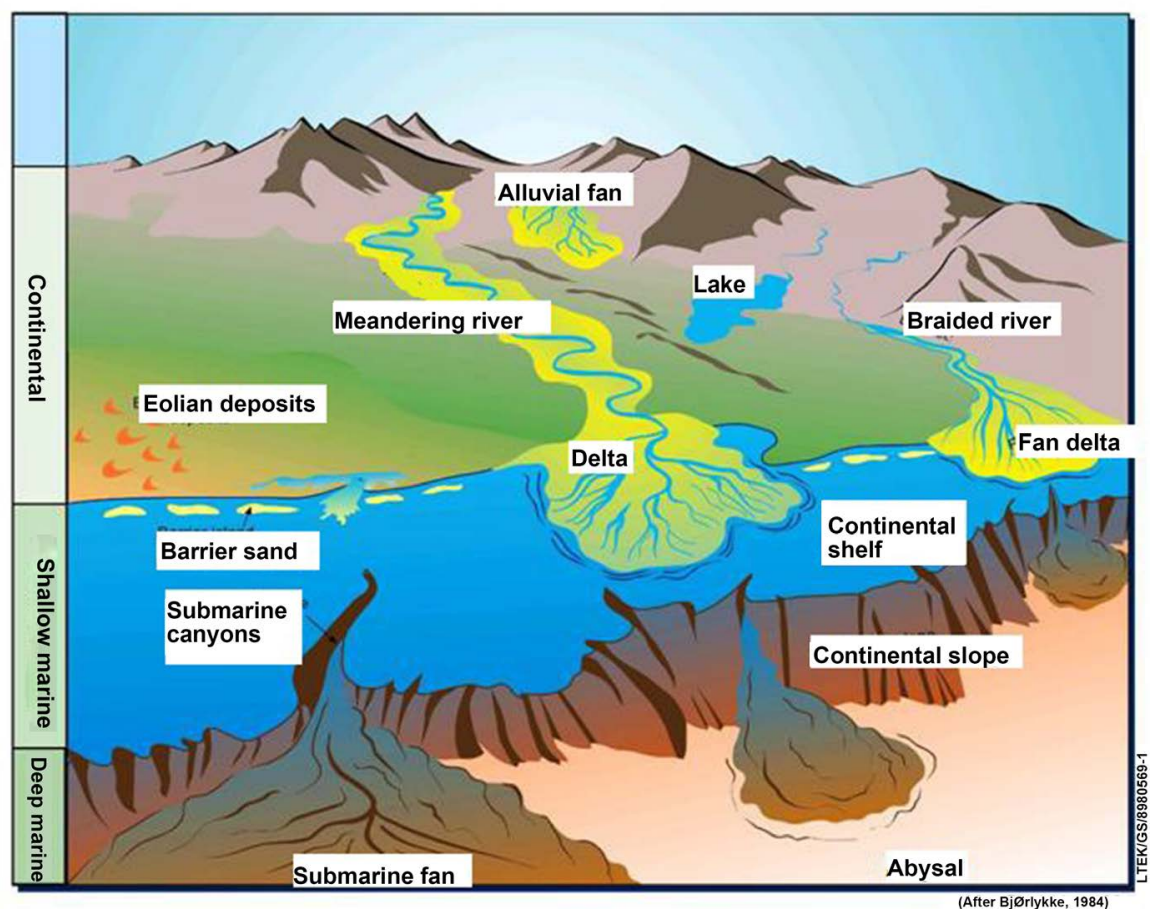

Figure 11. Depositional environment [16].

Table 4. Depositional features formed by rivers.

\begin{tabular}{cccc}
\hline Depositional features & Location & Energy level & Depositional space \\
\hline Delta & River mouth & Low & Large \\
Flood plains & Land adjacent to stream & Low & Intermediate \\
Levees & River bank & High & Small \\
Braided channels & Within river & Intermediate & Intermediate \\
Point bars & Meanders & Intermediate & Small \\
Alluvial fan & Base of desert & High (mouth) to low & Large \\
\hline
\end{tabular}

depositional environment is used to supplement subsurface data to reduce variogram uncertainty. In order to illustrate the importance of the knowledge of the depositional environment, two sets of models were constructed, the first set using subsurface well data only, and the second set using both well data and geological information. The reservoir models were built using the sequential approach work flow, where large scale properties are modeled first, followed by smaller uncertain properties.

\section{Methodology}

Geostatistical studies use hybrid simulation approaches that combine two or more conditional simulation techniques [17]. It involves first creating the structural design of the reservoir then the geological properties; beginning with facies then petrophysiscal properties [17]. In the structural design of the reservoir 
model, faults were first modeled and a grid network to accommodate the data was generated using the fault model. The setting for grid distance in I and J direction was set to 100 meters in I direction and 100 meters in J direction. Horizons and layers were modeled to characterize the internal structure of the model by building the vertical layering in the model. The vertical sequence of the layers mirrored the original lateral distribution of sedimentary environments and was guided by the interpreted depositional surfaces on seismic sections. The lithology facies were identified from well logs using alpha mapping method formula. The log analysis showed that the well penetrates a vertical sequence of sand and shale. This gave a picture of the environmental evolution at the time of deposition. It shows there were either low stand events when sedimentation was dominated by fluvial processes or high stand events when sedimentation was dominated by lacustrine processes.

Variograms were generated for prediction of facies both in the horizontal and vertical direction. The vertical variograms are presumed to be accurate due to the availability of continuous data along the well. The horizontal variograms are however presumed to be unreliable, since there is not enough data to statistically represent the information laterally. The variogram parameters; nugget and sill were obtained from the vertical variograms and were used for the horizontal variograms [17].

For illustration purposes, two sets of variograms were created to compare the results.

SET 1; Experimental variograms modeled from well data only.

SET 2; Experimental variograms modeled from well data and knowledge of the depositional environment. Using geological knowledge of the reservoir, parameter values were revised for the second set of models. A larger range was used in the horizontal direction as we presume that there is a large spatial continuity of the facies bodies due to the fact that the environment is a low energy environment with a large deposition space as shown in Table 4.

Each facies variograms was created individually in the vertical, major, and minor direction for each zone. Each zone was considered individually as it is discerned that the property values within the same zone have more depositional resemblence [17]. Once experimental variograms had been modelled, a mathematical variogram model was fitted to the experimental variogram points and used as input for modeling the facies model.

Only model variograms can be used by all the property modeling algorithms [18]. A spherical variogram was used for both cases. Using the variogram models, Sequential Indicator Simulation (SIS) algorithm conditioned to well data was used to simulate several realizations of the facies.

\section{Results and Discussion}

Based on sedimentary facies analysis on each well, two basic facies are defined for facies modelling; shale and sand. The two sets of facies models showed significant variation in the property distribution. Figure 12 shows the model 


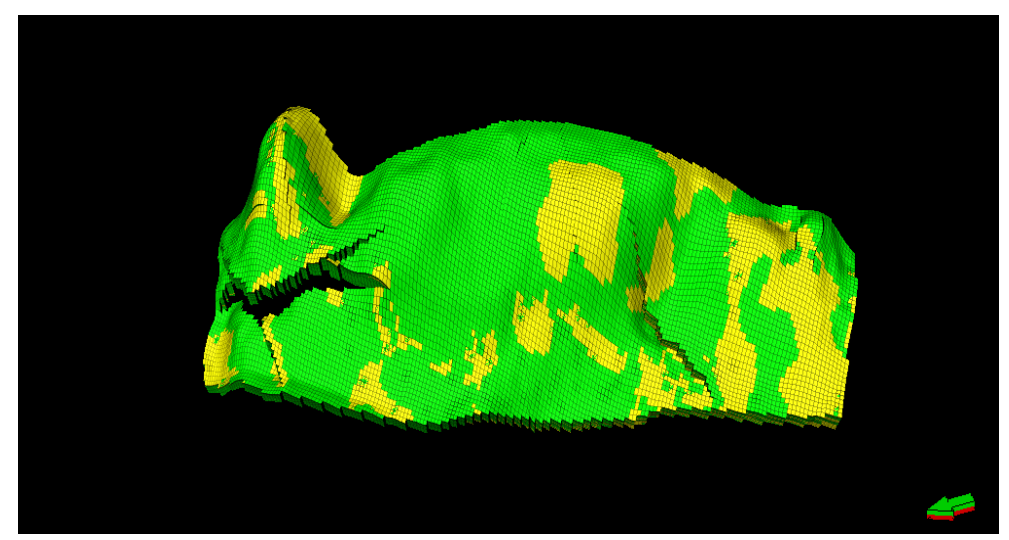

Figure 12. Facies Distribution based on well data variogram.

constructed without the influence of the depositional environment on the horizontal range. There is a random distribution of the facies, which does not depict the geological environment. Figure 13 shows the model constructed with the influence of the depositional environment on the horizontal range. This gave a realistic depiction of the geological environment of the reservoir. The distribution trend of the facies of set 2 resembled the proposed depositional model of the reservoir. Figure 14 shows the conceptual depositional model of the depositional environment. A slope setting with low energy level at the basin (lake) and a large deposition space. The river distributary channels are distributed to form a continuous sand body. The large sedimentation distribution space provided by the lake basin, accompanied with the low energy level, led to a larger range of continuity of the facies. This was confirmed by correlation within the wells. Well correlation gives a picture of the lateral variation of a facies body. The sand particles are deposited first as they are heavier and the shale particles are transported further on. The range, major and minor directions of the two facies were each derived from the depositional model and hence the resulting model had a closer resemblance to the depositional environment compared to the facies model created with only well data.

The accurate facies model will hence lead to accurate modeling of petrophysical properties and the geological model as a whole. This will lead to better forecasts and decision making using the reservoir model.

\section{Conclusions}

- Before modeling, a good comprehension of the depositional environment of the reservoir is necessary.

- The knowledge of the depositional environment in this paper was derived by studying the deposition space and energy level.

- Different depositional environments differ in terms of energy level and depositional space.

- The energy level at the time of deposition is not the only determinant in the continuity of the lithofacies. 


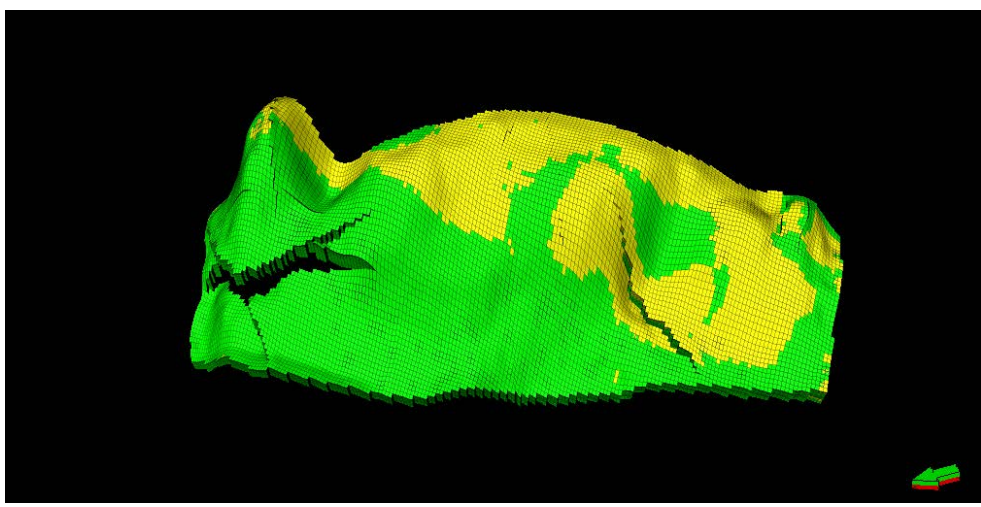

Figure 13. Facies Distribution based on well data and depositional environment knowledge variogram.

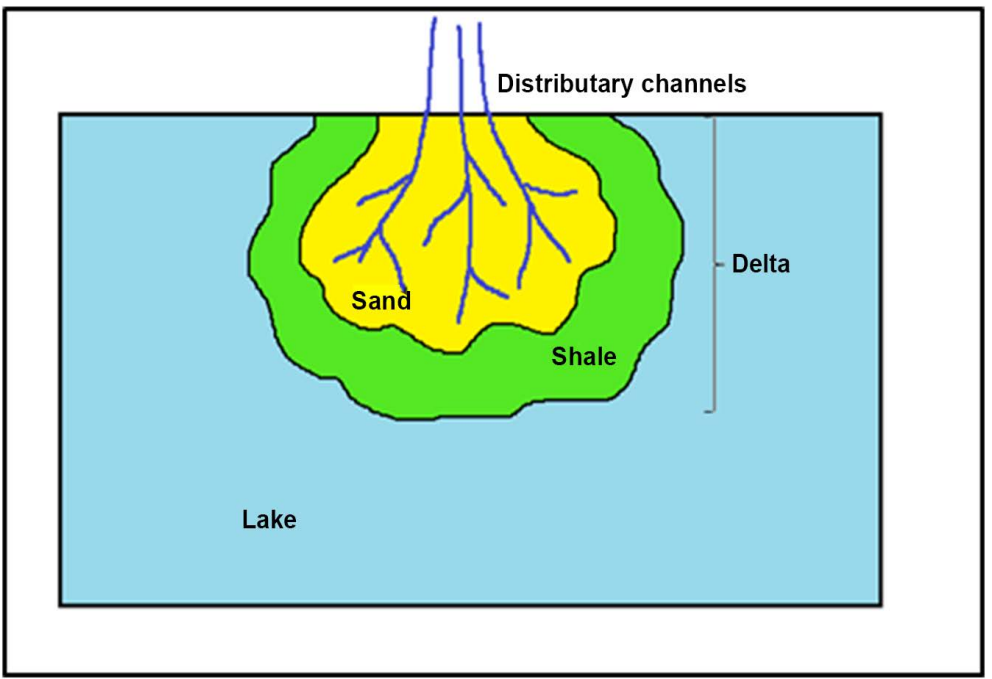

Figure 14. Proposed depositional environment.

- The knowledge of the depositional space is an important determinant as well in the continuity of the lithofacies and should be used together with the knowledge of the energy level.

- The knowledge of the depositional space and the energy level can be incorporated into the reservoir model through variograms. This helps us to know the range of the variograms.

- The methodology helped to produce a model that is similar to the geology of the reservoir, hence reducing uncertainty.

In comparison, the two sets of models showed significant variation in the property distribution. The first set gave a more random appearance of the facies distribution while the second set gave a more realistic depiction of the depositional environment of the reservoir.

\section{References}

[1] Gringarten, E. and Deutsch, C.V. (1999) Methodology for Variogram Interpretation and Modeling for Improved Reservoir Characterization. SPE Annual Technical 
Conference and Exhibition, Houston, Texas, 3-6 October 1999, SPE-56654-MS.

[2] Oloo, M.A. and Xie, C.J. (2018) Three-Dimensional Reservoir Modeling Using Stochastic Simulation, a Case Study of an East African Oil Field. International Journal of Geosciences, 9, 214-235. https://doi.org/10.4236/ijg.2018.94014

[3] Bahar, A., Ates, H., Kelkar, M. and Al-Deeb, M. (2001) Methodology to Incorporate Geological Knowledge in Variogram Modeling. SPE Asia Pacific Oil and Gas Conference and Exhibition, Jakarta, Indonesia, 17-19 April 2001, SPE-68704-MS.

[4] Deutsch, C.V. and Journel, A.G. (1998) GSLIB Geostatistical Software Library and User's Guide. In: Applied Geostatistics Series, Oxford University Press, New York.

[5] Grimes, D.I.F., Pardo-Igúzquiza, E. and Bonifacio, R. (1999) Optimal Areal Rainfall Estimation Using Raingauges and Satellite Data. Journal of Hydrology, 222, 93-108. https://doi.org/10.1016/S0022-1694(99)00092-X

[6] Margaret Oliver, R.W.J.G. (2009) Geostatistics in Physical Geography. The Royal Geographical Society, London.

[7] Cressie, N. and Hawkins, D.M. (1980) Robust Estimation of the Variogram. Journal of the International Association for Mathematical Geology, 12, 115-125. https://doi.org/10.1007/BF01035243

[8] Saeed Soltani-Mohammadi, M.S. (2016) A Simulated Annealing Based Optimization Algorithm for Automatic Variogram Model Fitting.

[9] Steele, R., Stephen, K. and Lin, X.-Q. (2013) Observed Spatial Statistics of Permeability and the Effects on Fluid Flow; Are We Getting It Right? EAGE Annual Conference \& Exhibition incorporating SPE Europec, London, UK, 10-13 June.

[10] Gribov, A., Krivoruchko, K. and Ver Hoef, J.M. (2005) Modified Weighted Least Squares Semivariogram and Covariance Model Fitting Algorithm. In: Yarus, J.M. and Chambers, R.L., Eds., Stochastic Modeling and Geostatistics, AAPG Computer Applications in Geology, Tulsa, Oklahoma.

[11] Mcbratney, A.B. and Webster, R. (1986) Choosing Functions for Semi-Variograms of Soil Properties and Fitting Them to Sampling Estimates. European Journal of Soil Science, 37, 617-639. https://doi.org/10.1111/j.1365-2389.1986.tb00392.x

[12] Introduction to Depositional Environments. https://www.slideshare.net/ay_arain39/deltas-13107361

[13] Sam Boggs, J. (2006) Principles of Sedimentology and Stratigraphy.

[14] Huggett, R.J. (2007) Fundamentals of Geomorphology.

[15] Miller, M.B. Alluvial Fans. http://www.seddepseq.co.uk/DEPOSITIONAL_ENV/Fluvial/Alluvial/AlluvialFan.h $\underline{\mathrm{tm}}$

[16] Statoil (2013) Depositional Environments. http://www.uio.no/studier/emner/matnat/math/MEK4450/h15/ppt/l1-2/5-depositio nal-environments-august-2015.pdf

[17] Gonzalez, R., Schepers, K., Reeves, S.R., Eslinger, E. and Back, T. (2008) Integrated Clustering/Geostatistical/Evolutionary Strategies Approach for 3D Reservoir Characterization and Assisted History-Matching in a Complex Carbonate Reservoir, SACROC Unit, Permian Basin. SPE 113978-PP.

[18] Schlumberger Petrel 2014 Software Manual. 